\title{
Transition from steady shear to oscillatory shear rheology of dense suspensions
}

\author{
Junhao Dong (1) and Martin Trulsson $\odot^{*}$ \\ Theoretical Chemistry, Lund University, 22100 Lund, Sweden
}

(Received 23 April 2020; accepted 20 October 2020; published 17 November 2020)

\begin{abstract}
Recent studies have highlighted that oscillatory and time-dependent shear flows might help increase the flowability of dense suspensions. While most focus has been on cross-flows we here study a simple two-dimensional suspensions where we apply simultaneously oscillatory and stationary shear along the same direction. We first show that the dissipative viscosities in this set-up significantly decrease with an increasing shear-rate magnitude of the oscillations and given that the oscillatory strain is small, in a similar fashion as found previously for cross-flow oscillations. As for cross-flow oscillations, the decrease can be attributed to the large decrease in the number of contacts and an altered microstructure as one transitions from a steady shear to an oscillatory shear dominated rheology. As subresults we find both an extension to the $\mu(J)$ rheology, a constitutive relationship between the shear stresses and the shear rate, valid for oscillatory shear flows and that shear-jamming of frictional particles at oscillatory shear dominated flows occurs at higher packing fractions compared to steady shear dominated flows.
\end{abstract}

DOI: 10.1103/PhysRevE.102.052605

\section{INTRODUCTION}

The flow of dense suspensions, even for the simplest systems, shows several nontrivial rheological behaviours such as shear-thinning [1], both continuous [2,3] and discontinuous shear-thickening [4-7], and shear jamming [8,9]. In the simplest models of a non-Brownian suspension composed of hard bodies the viscosity $\eta$ diverges as $\eta / \eta_{f} \sim\left(\phi_{c}-\phi\right)^{-\alpha}$, where $\eta_{f}$ is the solvent viscosity, $\phi$ the packing fraction, and $\alpha$ a critical exponent usually close to $2[10,11]$ and where $\phi_{c}$ depends on the friction [12], shape [13-19], and interactions [20,21]. The shear-thinning and shear-thickening can usually be attributed to a decreased or an increased importance of certain interactions compared to others. For example, shear-thinning of colloidal suspensions is due to a decreased importance of thermal collisional interactions (vibrations in "soft cages") compared to the shear stresses [22]. Shearthickening can be attributed to an increased importance of hydrodynamics $[6,23]$ or as in the case of discontinuous shearthickening the onset of frictional interactions above a certain stress threshold [4,5]. Shear-thickening can also be driven by inertial effects $[2,3]$ or a combination of the causes given above [24,25]. Acoustics [26] and oscillatory flows [27,28] have recently been shown to be effective in altering the flowability of suspension, where one has the ability to lower the the viscosity in a controlled manner (i.e., the resistance to flow). Especially cross-flows have turned out to be useful when an increased flowability of (almost) shear jammed dense sus-

\footnotetext{
*martin.trulsson@teokem.lu.se
}

Published by the American Physical Society under the terms of the Creative Commons Attribution 4.0 International license. Further distribution of this work must maintain attribution to the author(s) and the published article's title, journal citation, and DOI. Funded by Bibsam. pensions is desired and can even under certain circumstances unblock shear jammed suspensions [28]. The current understanding of this decrease in shear viscosity with cross-flows is based on a force chain tilting and eventual breakage of them as a consequence to this secondary oscillatory shear flow [27] and a random organisation [28,29], the later a concept closely related to reversibility of oscillatory shear flows for suspensions [29].

In this paper we show that by applying an oscillatory shear flow which is parallel to a stationary shear flow, one obtains an equivalent viscosity reduction as for cross-flow oscillations, in contradiction to previous findings [28]. By increasing the shear-rate magnitude of the oscillations, we find that the number of contacts per particle decreases as well as an altered microstructure compared to steady shear. These findings agrees well with previous findings for cross-flow oscillations and can be explained by the fact that the dense suspensions transit from a steady shear to an oscillatory shear rheology, where the latter has a significant lower viscosity at the same packing fraction and small oscillatory strains. We, furthermore, show that the shear-jamming point for a suspension composed of frictional particles shifts to higher packing fractions as oscillations with small strains are applied and approaches a new shear jamming packing fraction with a value just below the shear jamming point for a suspension composed of frictionless particles. For a suspension composed of frictionless particles we do not find any significant shift in the shear jamming point as oscillations are applied. We do, however, still notice a viscosity reduction, on the order of a magnitude, as oscillations are applied.

\section{STEADY-SHEAR AND OSCILLATORY-SHEAR RHEOLOGIES}

Consider a suspensions that is sheared with a timedependent shear rate $\dot{\gamma}=\dot{\gamma}_{0}+\dot{\gamma}_{1} \cos (\omega t+\delta)$, where $\dot{\gamma}_{0}$ is the stationary shear-rate, $\dot{\gamma}_{1}$ the shear-rate magnitude of the 
oscillatory shear, $\omega / 2 \pi$ the frequency of oscillatory shear, and $\delta$ a shift in time. Without any loss of generality, we assume from now on $\delta$ to be equal to zero and both $\dot{\gamma}_{1}$ and $\dot{\gamma}_{0}$ to be two positive numbers. While the viscosity in a steady shear flow $\left(\dot{\gamma}_{1}=0\right)$ is defined by a "stress" viscosity $\eta_{\sigma}=\langle\sigma\rangle /\langle\dot{\gamma}\rangle$, where $\sigma$ is the shear stress, it becomes obvious that this expression becomes imprecise and possibly invalid at pure oscillatory shear flows $\left(\dot{\gamma}_{0}=0\right)$ where $\langle\sigma\rangle \rightarrow 0$ and $\langle\dot{\gamma}\rangle \rightarrow 0$. For pure oscillatory shear flows one instead relies on the complex viscosity $\eta^{*}$. Assuming a pure viscous response, this complex viscosity $\eta^{*}$ becomes equal to its viscous part $\eta^{\prime}$ as $\eta^{*}=$ $\eta^{\prime}=\frac{\int_{0}^{2 \pi / \omega} \sigma(t) \cos (\omega t) d t}{\dot{\gamma}_{1} \int_{0}^{2 \pi / \omega} \cos ^{2}(\omega t) d t}=G^{\prime \prime} / \omega$, which is essentially a shearrate weighted quantity, with $G^{\prime \prime}=\frac{\omega^{2}}{\pi \dot{\gamma}_{1}} \int_{0}^{2 \pi / \omega} \sigma(t) \cos (\omega t) d t$ being the loss modulus [30]. We generalize this quantity and define shear-rate-averaged quantities as

$$
\langle A\rangle_{|\dot{\gamma}|}=\frac{\int_{0}^{2 \pi n / \omega}|A(t)||\dot{\gamma}(t)| d t}{\int_{0}^{2 \pi n / \omega}|\dot{\gamma}(t)| d t},
$$

where $A(t)$ is a time-dependent quantity of interest (e.g., shear stress $\sigma$ or number of contacts $Z$ ) and an integer $n$ (number of oscillation periods of the time-average). Shear-rate-averaged viscosities can then be calculated as $\eta_{|\dot{\gamma}|}=\langle\sigma\rangle_{|\dot{\gamma}|} /\langle\dot{\gamma}\rangle_{|\dot{\gamma}|}$, in perfect agreement with the viscous part of the complex viscosity for a pure oscillatory shear flow as well as the stress viscosity for a steady-shear flow. This new measure $\eta_{|\dot{\gamma}|}$, hence, offers a generalized way to measure viscosities, applicable to both steady-shear and oscillatory-shear flows as well as any combination of them. The power dissipated at any given time is equal to $\mathcal{D}(t)=\sigma(t) \dot{\gamma}(t)$. By normalizing the average power of dissipation $\langle\mathcal{D}(t)\rangle$ with $\left\langle\dot{\gamma}^{2}(t)\right\rangle$, which is related to the dissipation of an instantaneously relaxing Newtonian fluid, we obtain that $\langle\mathcal{D}(t)\rangle /\left\langle\dot{\gamma}^{2}(t)\right\rangle=\langle\sigma\rangle_{\mid \dot{\gamma}[} /\langle\dot{\gamma}\rangle_{|\dot{\gamma}|}$, i.e., our shear-rate-averaged viscosity is in fact a "dissipative" viscosity. For a Newtonian suspension with $\sigma=\eta^{S S}(\phi) \dot{\gamma}$ and an instantaneous relaxation of stresses in response to changes in shear-rate, the two viscosities, "stress" and "dissipative", yield the same values $\eta_{\sigma}=\eta_{|\dot{\gamma}|}=\eta^{S S}(\phi)$. However, for rapidly varying shear (i.e., unsteady) flow a suspension does not, in general, have sufficient time to restructure and adapt to the new conditions and one might easily obtain $\eta_{\sigma} \neq \eta_{|\dot{\gamma}|}$.

\section{SIMULATION METHOD AND DETAILS}

We consider suspensions composed of roughly 1000 nonBrownian polydisperse discs sheared between two rough walls, created by fusing particles together, at constant packing fractions or constant imposed pressure (see later). Particles interact via normal and tangential forces (frictional forces) with a Coulomb criteria for sliding where we set the particle friction coefficient $\mu_{p}$ equal to either 0.4 (frictional) or 0 (frictionless). The particles also interact with the solvent via hydrodynamic drag and torques, which are linear with the translational and angular velocity differences between the particle and fluid, respectively. The interstitial fluid velocity is described by a linear profile, $\mathbf{u}^{f}=(y \dot{\gamma}, 0)$, with the angular fluid velocity $\omega^{f}=\dot{\gamma} / 2$; where $y$ is the $y$ coordinate of the fluid and $\dot{\gamma}$ being the time-dependent shear rate we apply
TABLE I. Values of $\eta_{f} \dot{\gamma}_{\max } / k_{n}$ we have in our simulations at different $\phi$ 's for suspensions of frictional and frictionless particles.

\begin{tabular}{lllll}
\hline \hline & \multirow{2}{*}{ frictional } & & \multicolumn{2}{c}{ frictionless } \\
\cline { 5 - 5 }$\phi$ & $\eta_{f} \dot{\gamma}_{\max } / k_{n}$ & & $\phi$ & $\eta_{f} \dot{\gamma}_{\max } / k_{n}$ \\
\hline 0.67 & $\sim\left[10^{-6}, 10^{-5}\right]$ & & 0.72 & $\sim\left[10^{-6}, 10^{-5}\right]$ \\
0.72 & $\sim\left[10^{-6}, 10^{-5}\right]$ & & 0.74 & $\sim\left[10^{-7}, 10^{-5}\right]$ \\
0.74 & $\sim\left[10^{-7}, 10^{-6}\right]$ & & 0.76 & $\sim\left[10^{-7}, 10^{-5}\right]$ \\
0.76 & $\sim\left[10^{-7}, 10^{-6}\right]$ & & 0.79 & $\sim\left[10^{-7}, 10^{-5}\right]$ \\
0.79 & $\sim\left[10^{-7}, 10^{-6}\right]$ & & 0.82 & $\sim\left[10^{-8}, 10^{-6}\right]$ \\
\hline \hline
\end{tabular}

to the system. Both simulations with and without lubrication forces and torques were considered (see Appendix G for description of the lubrication forces). The particle dynamics were either Newtonian (for the cases with lubrication forces) or strictly overdamped (for the cases without lubrication forces). In the cases of the Newtonian dynamics we put the Stokes number $\left(S t=\frac{\rho \dot{\gamma} d^{2}}{\eta_{f}}\right.$, where $d$ is the average particle diameter and $\rho$ the mass density of the particles) smaller than 0.4 to ensure that we do not have non-negligible inertial effects. For our pressure controlled simulations we used the same dynamics for the two walls (moving as two blocks), in the normal direction, as for the freely flowing particles. For more details of the simulation model see [7,31].

For the simple system we are studying, we can identify three dimensionless parameters: $\mathcal{F}=\dot{\gamma}_{1} / \dot{\gamma}_{0}, \mathcal{G}=\dot{\gamma}_{1} / \omega$, and $\phi . \mathcal{G}$ gives here the maximum strain magnitude due to the oscillations. Parameters ranges studied are $\mathcal{F} \in\left[3 \times 10^{-2}, 3 \times\right.$ $\left.10^{2}\right]$ and $\mathcal{G} \in\left[10^{-2}, 10\right]$. In this work, we use the average particle diameter $d$ as the unit of length, $\eta_{f} / k_{n}$ as the unit of time, and $k_{n} d^{2}$ as the unit of energy. While our particles are soft (i.e., a harmonic potential between particles at contact), we obtain a quasihard core behavior by keeping the ratio $k_{n} / P>10^{4}$ (which is related to the overlap) at all times. Since $P \sim|\dot{\gamma}|$ and in order to ensure quasihard core condition, the maximum shear-rate $\dot{\gamma}_{\max }=\left|\dot{\gamma}_{0}\right|+\left|\dot{\gamma}_{1}\right|$ needs to be adjusted in units of $k_{n} / \eta_{f}$ for the various parameter combinations of $\phi, \mathcal{F}$, and $\mathcal{G}$, see Table I. The lower $\eta_{f} \dot{\gamma}_{\max } / k_{n}$ values corresponds in general to small $\mathcal{F}$ values and vice versa. For the pressure imposed simulations it is straightforward to keep $k_{n} / P \simeq 10^{4}$ as $P$ is now an input parameter. For the oscillatory shear flows we make sure that our time-step $\delta t$ was much smaller than the inverse frequency $2 \pi \omega^{-1}$ (i.e., $\omega \delta t \ll 1$ ). Naturally the same conditions were applied for the maximum shear-rates (i.e., $\dot{\gamma}_{\max } \delta t \ll 1$ ).

Simulations were typically carried out for a strain of at least 10 (i.e., $\int \dot{\gamma} d t>10$ ) and a minimum of ten oscillation periods $(n=10)$. Before starting to measure we pre-sheared all sampled for a few oscillatory periods and with a minimum of one absolute strain. We report both instantaneous time series of $\eta / \eta_{f}$ as well as shear-rate-averaged quantities [according to Eq. (1)] of $Z, \eta$, and later on also $\phi, J$ (viscous number), and $\mu$ (stress ratio). Viscosities are compared to the steady-shear viscosities $\eta^{S S}$ found for the same packing fractions of the system. 

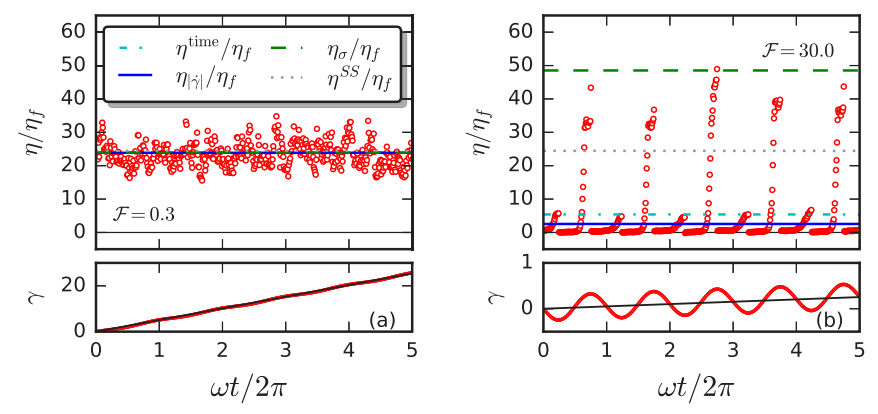

FIG. 1. Instantaneous relative viscosities at $\phi=0.67, \mu_{p}=0.4$, $\mathcal{G}=0.33$, and (a) $\mathcal{F}=0.3$, (b) $\mathcal{F}=30$. Lines give "stress" viscosity $\eta_{\sigma} / \eta_{f}$ (green-dashed), "dissipative" viscosity $\eta_{|\dot{\gamma}|} / \eta_{f}$ (solid-blue), and normal time-average $\eta^{\text {time }} / \eta_{f}$ (cyan-dash-dotted); grey dotted line indicates viscosity for steady shear (SS) flow at $\phi=0.67$ (i.e., $\mathcal{F}=0$ ), thin black solid line indicates zero line. The rectangular plots beneath the main figures illustrate how the strain evolves in the same time span for each corresponding case; black lines in the rectangular plots indicate how strain will evolve taking only account of the stationary shear-rate $\dot{\gamma}_{0}$.

\section{RESULT AND DISCUSSION}

\section{A. Instantaneous viscosities}

Figure 1 shows two typical cases of how the instantaneous viscosities varies with time at two different oscillation amplitudes. When $\mathcal{F}<1$ (small to moderate shear-rate oscillations compared to the average shear flow) the instantaneous viscosity only mildly fluctuates around an average viscosity equal to that of $\eta^{S S}(\phi)$ seen in steady state [2], see Fig. 1(a). However, when $\mathcal{F}>1$ (large shear-rate oscillations compared to the stationary shear flow) two distinct and alternating peaks appear with each period separated by zones with almost zero instantaneous viscosities, see Fig. 1(b). These peaks correlate well with the peaks in the strain with a maximum just before shear-reversal. One sees that the measure $\eta_{\sigma}$ poorly represents a suspension's instantaneous viscosity and is closer to the suspension's peaks in instantaneous viscosity at large oscillations [see Fig. 1(b)]. In general $\eta_{|\dot{\gamma}|}$ performs better in representing a suspension's instantaneous viscosity and is closer to a brute-force time-averaged viscosity $\eta^{\text {time }}=$ $\omega \int_{0}^{2 \pi n / \omega} \eta(t) d t /(2 \pi n)$. Similar alternating peaks are seen also in the instantaneous stresses (shear stress and pressure) both at this packing fraction and higher ones and as soon as $\mathcal{F}$ is large, see Appendix A.

\section{B. "Dissipative" viscosity at constant packing fraction}

In Fig. 2 we show how (a) the "dissipative" viscosities $\eta_{|\dot{\gamma}|} / \eta^{S S}$, (b) the number of contacts $Z_{|\dot{\gamma}|}$, the average dissipation rate $\mathcal{D}$ normalized by (c) $\eta^{S S} \dot{\gamma}_{0}^{2}$ or (d) $\eta^{S S} \dot{\gamma}_{1}^{2}$ vary with $\mathcal{F}$ at various oscillating strains $\mathcal{G}$, at $\phi=0.76$ (results for suspensions at other $\phi$ 's as well as for frictionless suspensions at $\phi=0.76$ can be found in the Appendix D). For all $\mathcal{G}$ the viscosities are close to $\eta^{S S}(\phi)$ as soon as $\mathcal{F}<1$ in agreement with previously reported results using cross-flow oscillations [28]. At $\mathcal{F}>1$ the viscosities decrease, moderately for large $\mathcal{G}$ and substantially for small $\mathcal{G}$ (i.e., small strain amplitude). This also illustrated in Fig. 3 where we
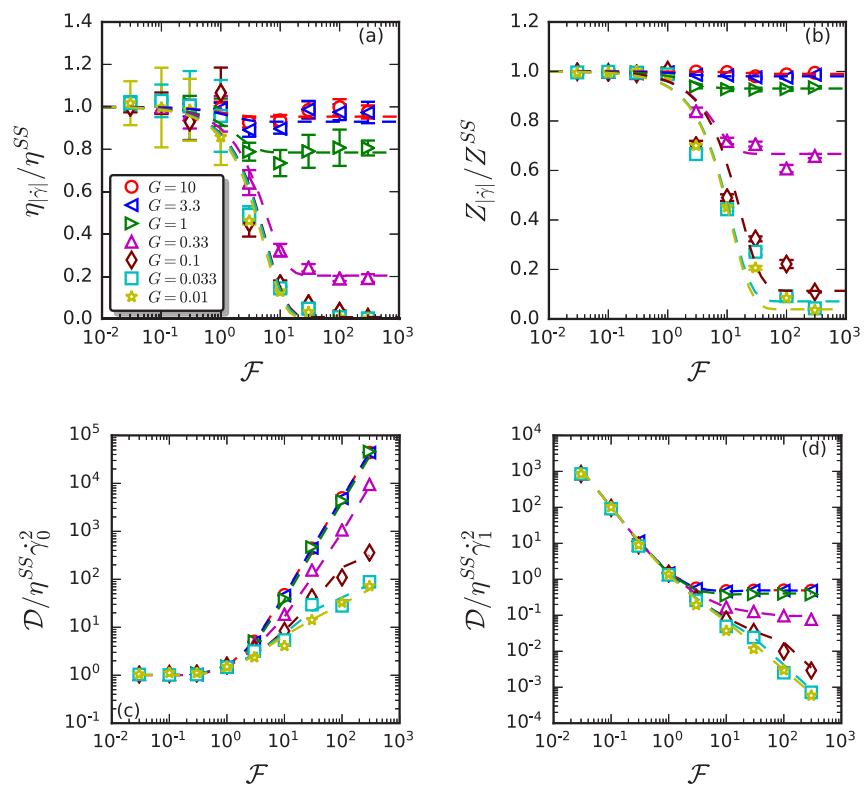

FIG. 2. (a) Reduced viscosity $\eta_{|\dot{\gamma}|} / \eta^{S S}$, (b) $Z_{|\dot{\gamma}|}$, (c) $\mathcal{D} / \dot{\gamma}_{0}^{2}$, and (d) $\mathcal{D} / \dot{\gamma}_{1}^{2}$ as function of $\mathcal{F}$ at various $\mathcal{G}$, at $\phi=0.76$ and $\mu_{p}=0.4$; dashed lines in (a) and (b) are best fits using a phenomenological hyperbolic tangent function $A_{|\dot{\gamma}|} / A^{S S}=1-c_{1} \tanh \left(c_{2} \mathcal{F}\right)$, where $c_{1}=\left(1-A_{|\dot{\gamma}|}^{\mathcal{F}=\infty} / A^{S S}\right)$ and $c_{2}$ are two free parameters and $A$ are either $\eta_{|\dot{\gamma}|} / \eta_{f}$ or $Z_{|\dot{\gamma}|}$, and dashed lines in (c) and (d) are $\eta_{|\dot{\gamma}|} / \eta^{S S}(1+$ $\left.0.5 \mathcal{F}^{2}\right)$ and $\eta_{|\dot{\gamma}|} / \eta^{S S}\left(\frac{1}{\mathcal{F}^{2}}+0.5\right)$ with $\eta_{|\dot{\gamma}|}$ obtained from (a).

show how the reduced viscosity $\eta_{|\dot{\gamma}|} / \eta^{S S}$ at $\mathcal{F}=\infty$ varies with $\mathcal{G}$. For large $\mathcal{G}$ the viscosity is close to $\eta^{S S}$ but does significant decrease as $\mathcal{G}$ is lowered. This effect seems to only be mildly affected by the packing fraction. The "normal" viscosity $\eta_{|\dot{\gamma}|}^{P}=\langle P\rangle_{|\dot{\gamma}|} /\langle\dot{\gamma}\rangle_{|\dot{\gamma}|}$ behaves essentially the same for the "dissipative" viscosity [see Fig. 2(a)], as shown in the Appendix C. The same trends are also found in the number of contacts, highlighting that the former is a consequence of the later. In particular, we see that the viscosity and the number of contacts both decrease to zero at high values of $\mathcal{F}$ and low values of $\mathcal{G}$. We interpret this as being in metareversible (finite $\mathcal{F}$ ) or reversible states $(\mathcal{F}=\infty)$ as has previously been observed for pure oscillatory shear flows of suspensions [29,32,33]. Metareversible is in where the states are only

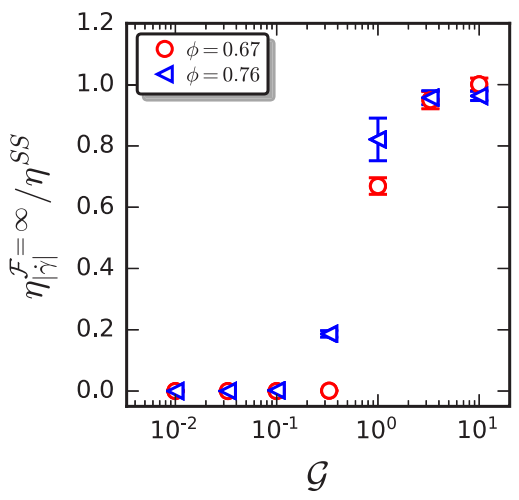

FIG. 3. Rescaled viscosity $\eta_{|\dot{\gamma}|} / \eta^{S S}$ at $\mathcal{F}=\infty$ as functions of $\mathcal{G}$ with $\mu_{p}=0.4$ and $\phi=0.67$ or 0.76 as indicated in the legend. 

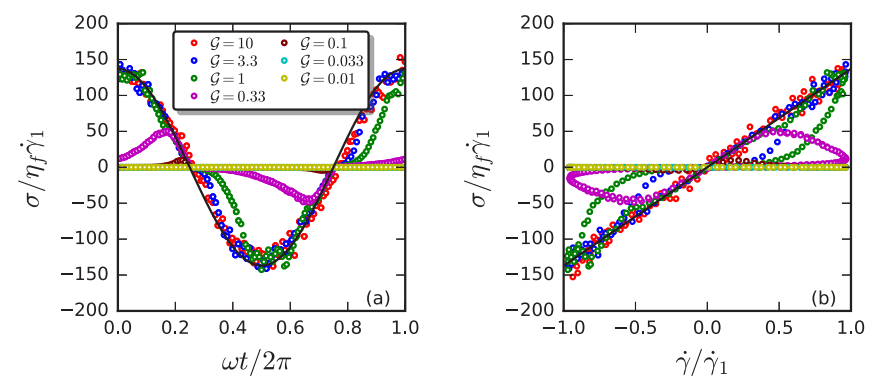

FIG. 4. (a) Time series of rescaled shear stress $\sigma / \eta_{f} \dot{\gamma}_{1}$ and (b) corresponding Lissajous curves of oscillatory flows at various $\mathcal{G}, \phi=0.76, \mathcal{F}=\infty$, and $\mu_{p}=0.4$; black solid lines in (a) and (b) are theoretical predictions assuming that a suspension follows constitutive relationships obtained from steady shear, i.e., $\sigma=\eta^{S S} \dot{\gamma}$.

reversible for a certain time and will eventually be broken due to the stationary shear flow. Figure 4 shows (a) time series of the rescaled shear stress $\sigma / \eta_{f} \dot{\gamma}_{1}$ and (b) the corresponding Lissajous curves for the frictional suspensions at $\mathcal{F}=\infty$ and various $\mathcal{G}$ at $\phi=0.76$, i.e., the asymptotic cases of Fig. 2 . The black lines are theoretical predictions for an instantaneous relaxing suspension following a steady-shear rheology, i.e., $\sigma=\eta^{S S} \dot{\gamma}$, at pure oscillatory shear flows. At $\mathcal{G} \geqslant 3.3$, where the viscosities have only a negligible decrease, the time series show an almost instantaneous response to $\dot{\gamma}$ and the corresponding Lissajous curves are linear with only tiny fluctuations. As $\mathcal{G}$ decreases, both the time series and the Lissajous curves deviate from the steady shear with deviations that are increasing as $\mathcal{G}$ is lowered. At $\mathcal{G}=0.033$ and 0.01 where the "dissipative" viscosities have reached zero, the shear stresses are constantly zero in the Lissajous curves as expected.

Our results are hence in line with what has been previously found for cross-flows oscillations [27,28], but here we clearly show that even oscillatory flows which are parallel to the average shear flow are sufficient for increasing flowability (i.e., lowering viscosity). This puts new doubt on the explanation that the increasing flowability is due to that the cross flow introduces a orthogonal perturbation which tilt the force chains (and if strong enough break them), as there is no possibility for such orthogonal perturbations in two dimensions. We here instead show that parallel oscillations decrease the number of contacts per particle and, hence, reorganize the particles microstructure (including breakage of force chains). Figure 5 shows two collapses (one for frictional particles and the other for frictionless) of rescaled viscosities $\eta_{|\dot{\gamma}|} / \eta_{f}$ versus the number of contact $Z_{|\dot{\gamma}|}$ for various $\phi, \mathcal{F}$, and $\mathcal{G}$, highlighting the strong correlation between these quantities. Such observation is in line with what has been reported previously [28]. As a note, using the shear-rate-weighted measure for cross-flows would leave previous results unaltered as they were measured along a direction where the shear-rate was constant in time. For a comparison between "dissipative" and "stress" viscosities in with shear oscillations in the parallel direction see the Appendix B. While results presented in the main text of this paper are without any lubrication forces, we found similar results with lubrification forces included (see Figs. 17-18 in the Appendices). The main difference is that
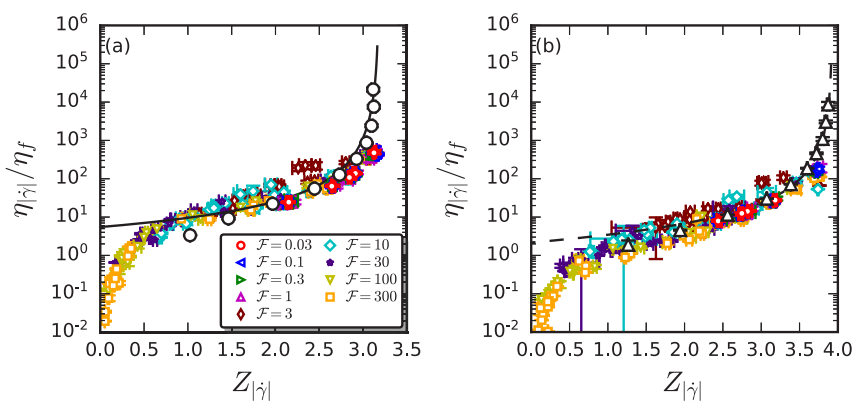

FIG. 5. Rescaled viscosities $\eta_{|\dot{y}|} / \eta_{f}$ as functions of the number of contacts per particle $Z_{|\dot{\gamma}|}$ for (a) frictional suspensions and (b) frictionless suspensions. Black symbols corresponds to steady-shear values $(\mathcal{F}=0)$ [25] for (circles) frictional and (triangles) frictionless particles, lines according to the constitutive laws for steady-shear, see [25]. Various symbols corresponds to various $\mathcal{F}$ as indicated in the legends, with $\phi \in[0.67,0.79]$ for frictional and $\phi \in[0.72,0.82]$ for frictionless particles and $\mathcal{G} \in\left[10^{-2}, 10\right]$.

the relative viscosities reach higher (finite) plateau values at large $\mathcal{F}$ and small $\mathcal{G}$ values. The viscosity reduction can hence be understood as a transition from a steady shear rheology to oscillatory shear one where the latter one has lower number of contacts per particles (and hence less force chains in general) at the same packing fraction and small oscillatory strains. While cross-flow oscillations has the possibility to tilt and break force chains, parallel-flow oscillations only has the possibility to break chains by shear-reversals which in its own would create new force chains (along the new and orthogonal compression axis). Hence, there is a conceptual difference between cross-flow and parallel-flow oscillations. In the force chain picture one would not expect a lowered viscosity (or at a maximum halved viscosity) when applying parallel oscillations around a stationary flow. We see a substantial larger viscosity reduction which instead seems to be related to a random organisation mechanism $[28,29]$ and a nonsliding of frictional contacts.

As discussed by Ness et al. [28] the dissipation per strain might be a more interesting quantity, especially for several industrial applications where one wants the lower the energy consumption per strain. Looking at this quantity ${ }^{1}$, see Fig. 2(c), one finds that the relative dissipation per strain remains equal to that at steady-shear at low $\mathcal{F}$ whereas it increases as $\mathcal{F}>1$. The increase at large $\mathcal{F}$ is in agreement with cross-flow results [28]. In general, one finds that $\mathcal{D} / \dot{\gamma}_{0}^{2}=$ $\eta_{|\dot{\gamma}|}\left(1+0.5 \mathcal{F}^{2}\right)$ and $\mathcal{D} / \dot{\gamma}_{1}^{2}=\eta_{|\dot{\gamma}|}\left(1 / \mathcal{F}^{2}+0.5\right)$. The scaling of $\mathcal{D}$ with respect to $\mathcal{F}$ is derived by having $\mathcal{D}=\eta_{|\dot{\gamma}|}\left\langle\dot{\gamma}(t)^{2}\right\rangle$. With $\dot{\gamma}(t)=\dot{\gamma}_{0}+\dot{\gamma}_{1} \cos (\omega t),\left\langle\dot{\gamma}(t)^{2}\right\rangle=\dot{\gamma}_{0}^{2}+0.5 \dot{\gamma}_{1}^{2}$. Therefore, $\mathcal{D} / \dot{\gamma}_{0}^{2} \sim\left(1+0.5 \mathcal{F}^{2}\right)$ and $\mathcal{D} / \dot{\gamma}_{1}^{2} \sim\left(1 / \mathcal{F}^{2}+0.5\right)$. The quantity $\mathcal{D} / \dot{\gamma}_{1}^{2}=\eta_{|\dot{\gamma}|}\left(1 / \mathcal{F}^{2}+0.5\right)$ might be more interesting for flows which are almost purely oscillatory shear flows. Notice that $\eta_{|\dot{\gamma}|}$ is itself dependent on $\mathcal{F}$, as well as $\mathcal{G}$ and $\phi$. While we get a monotonic relative increase for the rate of dissipation both from (c) a pure steady shear flow and (d) a pure oscillatory shear flow, cross-flows seems to show a

\footnotetext{
${ }^{1}$ The dissipation per strain in the primary direction is given by $\mathcal{W}=$ $\langle\mathcal{D}\rangle / \dot{\gamma}_{0}$ with $\mathcal{W}_{\mathcal{S S}}=\eta^{S S} \dot{\gamma}_{0}^{2} / \dot{\gamma}_{0}$ for steady-shear conditions.
} 

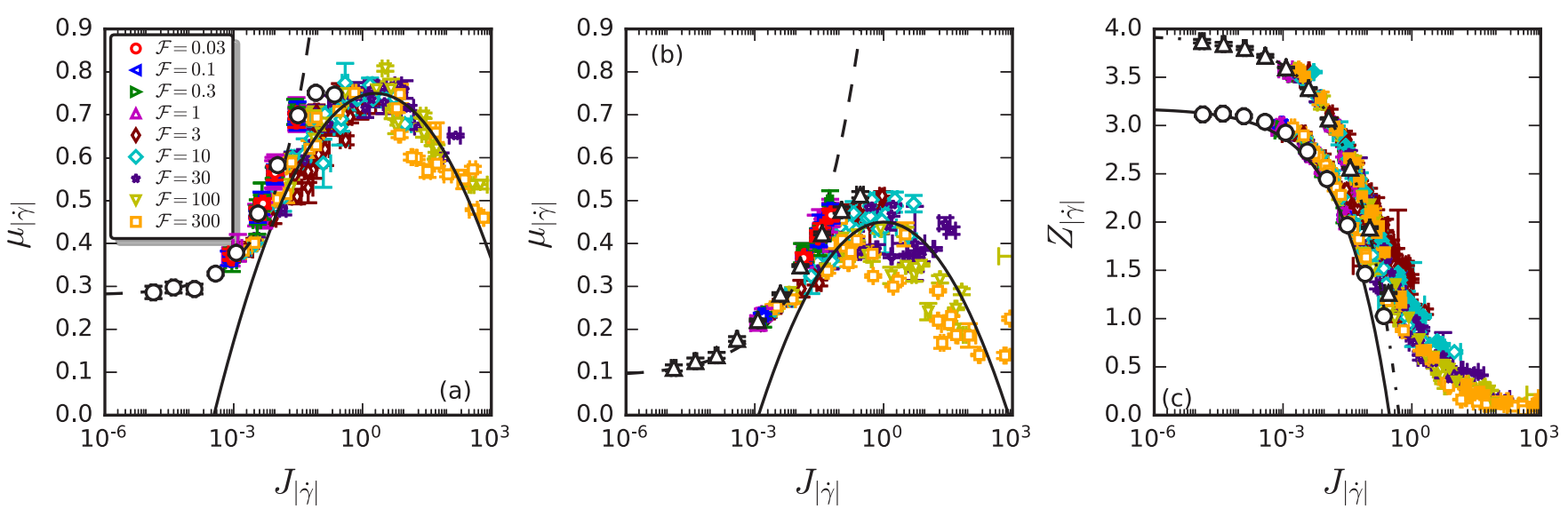

FIG. 6. Flow curves with extended $\mu(J)$ rheology for (a) $\mu$ for suspensions with frictional particles $\left(\mu_{p}=0.4\right)$, (b) $\mu$ for suspensions with frictionless particles $\left(\mu_{p}=0\right)$, and (c) $Z$ for both suspensions composed of either frictional (empty symbols) or frictionless particles (filled symbols). Black symbols corresponds to steady-shear values $(\mathcal{F}=0)$ [25] for (circles) frictional and (triangles) frictionless particles, lines according to [25]. Various symbols corresponds to various $\mathcal{F}$ as indicated in the legends, with $\phi \in[0.67,0.79]$ for frictional and $\phi \in$ $[0.72,0.82]$ for frictionless particles and $\mathcal{G} \in\left[10^{-2}, 10\right]$.

modest nonmonotonicity [28]. This discrepancy could possibly be attributed to that we neglected hydrodynamic interactions between pairs of particles or indeed that having the oscillations perpendicular to the average flow is slightly more beneficial in regards to lowering the dissipation per strain. However, we did not see any nonmonotonic for simulations with lubrication interaction included (see Appendix G), making the latter a more likely cause. An important finding from above is that $\eta_{|\dot{\gamma}|, \mathcal{F}=\infty} \leqslant \eta_{|\dot{\gamma}|, \mathcal{F}=0}=\eta^{S S}(\phi)$ for the same $\phi$, where viscosities for oscillatory flows and steady state differ for small to moderate oscillatory strains $(\mathcal{G}<3.3)$, hence do not respect the Cox-Merz rule, and are approximately equal otherwise. Interestingly, there exists a small regime $(1<\mathcal{F}<$ 10) for which the viscosities are lower both that of steady shear flow and oscillatory shear flow viscosities for the largest oscillatory strains [see Fig. 2(a)].

Suspension rheology can be reformulated using a viscous number $J=\eta_{f} \dot{\gamma} / P$, where $\phi(J), \mu(J)$, and $Z(J)$ all are constitutive relationships and functions of only $J$ in the hard-body limit $[2,11]$, where $P$ is the pressure and $\mu=\sigma / P$ the stress ratio. We here show that one can expand this formulation to oscillatory flows by using a shear-rate-weighted viscous number $\boldsymbol{J}_{|\dot{\gamma}|}=\eta_{f}\langle\dot{\gamma} / P\rangle_{|\dot{\gamma}|}$, where $P$ is measured in the center of the cell. By doing so we find a collapse of $\mu_{|\dot{\gamma}|}\left(J_{|\dot{\gamma}|}\right)$ and $Z_{|\dot{\gamma}|}\left(J_{|\dot{\gamma}|}\right)$ as soon as $\mathcal{F} \gg 1$ for a large parameter space of $\phi$ and $\mathcal{G}$, see Fig. 5, with a crossover between steadyshear and oscillatory dominated flows around $\mathcal{F} \sim 1$. Data points for which $\mathcal{F} \ll 1$ are all well captured by the original $\mu(J)$ rheology whereas data points for which $\mathcal{F} \gg 1$, and with $J_{|\dot{\gamma}|} \gg J^{S S}(\phi)$, are better described by the empirical relation $\mu_{|\dot{\gamma}|} \simeq \mu^{\max }-\kappa\left[\ln \left(J_{|\dot{\gamma}|}\right)-\ln \left(J_{|\dot{\gamma}|, 0}\right)\right]^{2}$ with $\mu^{\max } \simeq$ $0.75, J_{|\dot{\gamma}|, 0} \simeq 2$, and $\kappa=0.01$, shown in Fig. 6 (a) as a black solid line. A similar trend can be found for suspensions composed of frictionless particles, even though the collapse is slightly worse, as seen in Fig. 6(b). Here, the black solid line is the same constitutive law as in Fig. 6(a) but with $\mu^{\max } \simeq$ $0.45, J_{|\dot{\gamma}|, 0} \simeq 1$, and $\kappa=0.01$. The collapse works slightly better considering $Z_{|\dot{\gamma}|}$ plotted against $J_{|\dot{\gamma}|}$, see Fig. 6(c). An alternative and slightly worse definition of $J_{|\dot{\gamma}|}$ can be found in the Appendix E.

\section{Shift of jamming packing fraction}

We now explore if one can cross the shear-jamming packing fraction by having shear flow oscillations. We achieve this by doing pressure imposed simulations rather than constant volume. This replaces the control parameter $\phi$ by $J_{|\dot{\gamma}|}$. We study the asymptotic cases where $\mathcal{F}=\infty$ (having $\dot{\gamma}_{0}=0$ ), and $\boldsymbol{J}_{|\dot{\gamma}|} \in\left[10^{-5}, 10^{1}\right]$ keeping the oscillatory strains $\mathcal{G}$ fixed, which is achieved by varying $\dot{\gamma}_{1}$ and $\omega$ simultaneously. Indeed, as observed by cross-flow oscillations we find that the shearjamming point shifts to higher packing fraction compared to steady-shear for flows composed of frictional particles. For $\mathcal{F}=\infty$ there is no difference between if the flow are
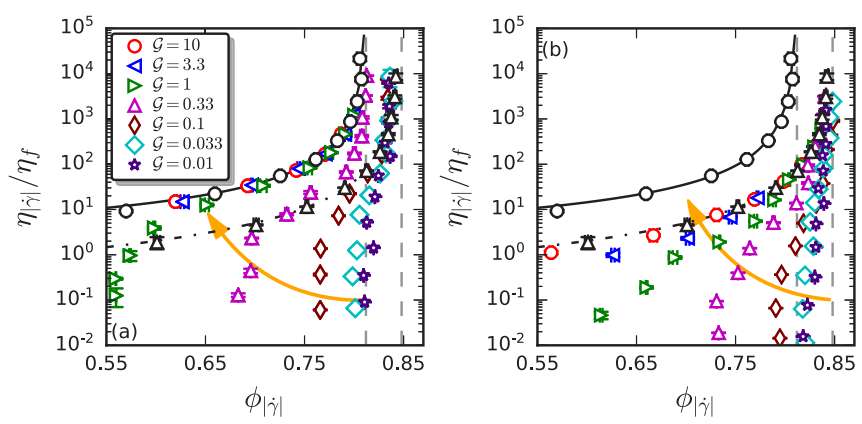

FIG. 7. Reduced viscosity as function of $\phi$ at various $\mathcal{G}$ and $\mathcal{F}=$ $\infty$ (pure oscillatory flows). Suspension composed of (a) frictional and (b) frictionless particles. Black symbols correspond to steadyshear viscosities for (circles) frictional and (triangles) frictionless, taken from [25]. The black lines corresponds to best fits according to $\eta(\phi) / \eta_{f}=a\left(\phi_{c}^{S S}-\phi\right)^{-\alpha}$ for (solid) frictional and (dash-dotted) frictionless particles. Vertical grey dashed lines show the locations of the corresponding shear-jamming points, with and $\phi_{c}^{S S \text {, frictionless }=}$ $0.848 \pm 0.002$ and $\phi_{c}^{S S \text {, frictional }}=0.812 \pm 0.002$. Orange arrows indicate the increase of $\mathcal{G}$. 

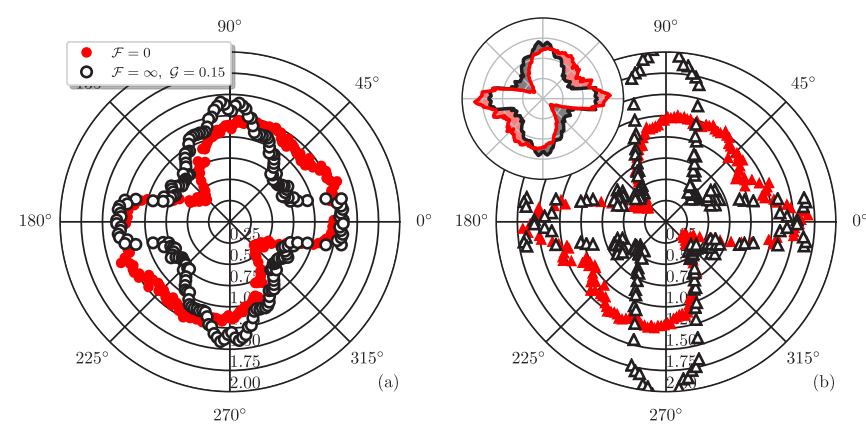

FIG. 8. Normalized polar contact probability functions of the particles at $\phi=0.79$. (a) Steady shear with $\eta_{|\dot{\gamma}|} / \eta_{f}=451$ (red filled circle) and oscillatory shear with $\eta_{|\dot{\gamma}|} / \eta_{f}=29$ (black empty circle) of frictional particles, (b) steady shear of frictionless particles with $\eta_{|\dot{y}|} / \eta_{f}=26$ (red filled triangle) and oscillatory shear with $\eta_{|\dot{\mid}|} / \eta_{f}=$ 2.5 (black empty triangle). The inset in (b) shows the comparison between the frictional case under oscillatory shear (black line) and the frictionless case under steady shear (red line); the shaded region indicates the difference between two lines, with the red region implies the region where the red line has values larger than the black one, and the black region the opposite.

cross-flow or parallel-flow as such distinction is ill-defined, highlighting that it is not cross-flow per see that leads to the shift in shear-jamming points but the fact that the flow is dominated by the oscillatory shear flow behavior. Figure 7 shows the viscosity at various fixed oscillatory strains. The viscosities follow the steady-shear viscosities for the corresponding system as soon as the oscillatory strain $\mathcal{G}$ is large. As the oscillatory strain is lowered the viscosities of suspensions composed of frictional particles start to follow the viscosity branch corresponding to suspensions composed of frictionless particles at steady shear state in a narrow regime close to the frictionless shear jamming point. In the Appendix F, we show some time series of $\phi$ close to shear-jamming packing fractions (at $J_{|\dot{\gamma}|} \sim 10^{-3}$ ) at $\mathcal{G}=0.33$ and 0.01 for both the frictional and frictionless cases. We see that for all the cases shown, $\phi$ only show minor fluctuation around their average values, with about $1 \%$ of relative fluctuation at $\mathcal{G}=0.33$ and almost negligible fluctuations $(\ll 0.1 \%)$ at $\mathcal{G}=0.01$. This new and partially unexplored jamming point for oscillatory flows which, as we denote $\phi_{c}^{O S}$ as compared to the steady state jamming point $\phi_{c}^{S S}$, will hence be dependent on $\mathcal{G}$ with $\phi_{c}^{O S \text {,frictional }} \rightarrow \phi_{c}^{S S \text {,frictional }}$ as $\mathcal{G} \rightarrow \infty$ and $\phi_{c}^{O S \text {,frictional }} \rightarrow$ $0.835<\phi_{c}^{S S \text {, frictionless }}$ as $\mathcal{G} \rightarrow 0$. The transition is found to occur around $\mathcal{G}=0.1$. We do not yet know if this transition is continuous or discontinuous. We do however observe that the viscosity is substantially lowered even before a shift in shearjamming packing occurs [see $\mathcal{G}=0.33$ markers in Fig. 7(a)].

For suspensions composed of frictionless particles the shear-jamming point seems not to shift for pure oscillatory

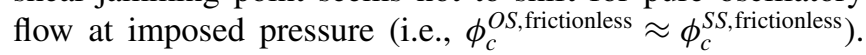
The viscosities do, however, decrease by roughly one order of magnitude. Our results are in agreement with previous findings for oscillatory cross-flows [28] for low $\mathcal{G}$ with the exception that we also find an increased flowability also for frictionless particles at oscillatory flows and low $\mathcal{G}$.

\section{Altering in microstructure}

As a final test we study the microstructure of steadyshear, oscillatory shear flow samples for both frictional and frictionless at constant packing fraction $\phi=0.79$. Figure 8 shows how the contact distribution changes from a twofold rotational symmetry for steady shear flows to having a fourfold symmetry for oscillatory shear flows. Even if the pure oscillatory flow of the frictional particles [black empty circles in (a)] has a viscosity similar to that of a frictionless sample at the same packing fraction [red filled triangles in (b)] the two microstructures are clearly different, as seen in the inset of Fig. 8(b), indicating that the "mechanism" for jamming is not the same in the two approaches and the collapse of the oscillatory viscosities at low strains onto the steady-shear frictionless branch is probably fortuitous.

\section{CONCLUSION}

In this paper we have shown that (i) oscillatory shear flows parallel to the average flow leads to a decrease in viscosities, (ii) the $\mu(J)$ rheology can be extended to oscillatory shear flows, and (iii) the oscillatory shear-jamming packing fraction is unaltered compared to steady shear for frictionless particles but shifted upwards for frictional particles. Our understanding of why this shift occurs in the frictional but not in the frictionless case is that for oscillatory flows with small strains (less than 0.1) the tangential springs do not have time to get enough strained to mechanically stabilize the suspensions. Frictionless particles lacks this possibility altogether and are hence unaffected by this effect. Our overall understanding of the viscosity reduction of dense suspensions is that it should be regarded as a transition from a steady shear flow rheology to a oscillatory shear rheology, where the latter has a lower viscosity at low oscillatory strains compared to the former at the same packing fraction. Hence, this opens up for the alternative strategy of using shear oscillations along a stationary shear direction (or any other chosen direction) to unblock shear jammed dense suspensions of frictional particles and/or facilitate flow.

It would be fruitful to expand the $\mu(J)$ rheology to granular rheology in line with Ref. [34] and using the inertial number $I$ instead of $J$, to explore the role of nonlocal rheology [35], and study linear combinations of shear oscillations perpendicular to each other with or without a stationary shear flow.

\section{ACKNOWLEDGMENTS}

M.T. acknowledges financial support by the Crafoord Foundation (20190650). The simulations were performed on resources provided by the Swedish National Infrastructure for Computing (SNIC) at the center for scientific and technical computing at Lund University (LUNARC).

\section{APPENDIX A: INSTANTANEOUS VISCOSITIES, STRESSES, AND PRESSURES}

In Fig. 9 we show the instantaneous stress and pressure at $\phi=0.67, \mu_{p}=0.4, \mathcal{G}=0.33$, and $\mathcal{F}=30$ [i.e., for the same case as in Fig. 1(b) in the main text] as function of time. Similar as for the instantaneous viscosity [see Fig. 1(b) 

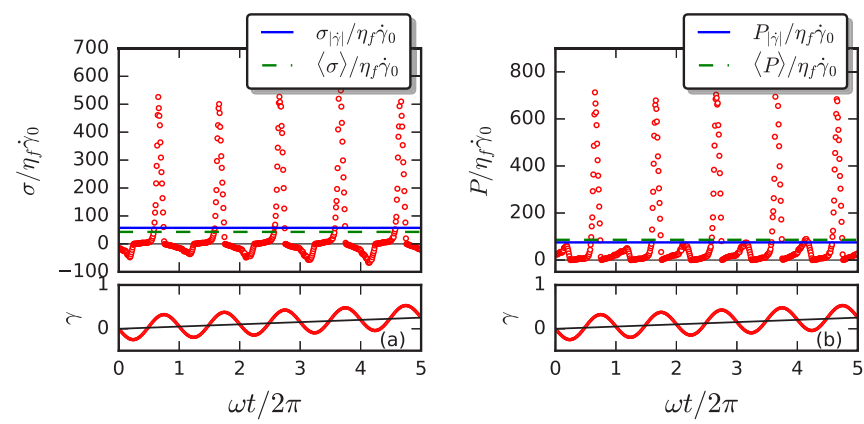

FIG. 9. (a) The instantaneous rescaled stress $\sigma / \eta_{f} \dot{\gamma}_{0}$ and (b) the instantaneous rescaled pressure $P / \eta_{f} \dot{\gamma}_{0}$ at $\phi=0.67, \mu_{p}=0.4, \mathcal{G}=$ 0.33 , and $\mathcal{F}=30$ [i.e., the same case as in Fig. 1(b) in the main text] as function of time. Green-dashed lines indicate values of $\langle\sigma\rangle / \eta_{f} \dot{\gamma}_{0}$ and $\langle P\rangle / \eta_{f} \dot{\gamma}_{0}$, respectively, and blue solid lines indicate the corresponding shear-rate-averaged quantities. The rectangular plots beneath the main plots illustrate how strain evolves in the same time span with black lines indicate how strain will evolve accounting only for the average shear-rate $\dot{\gamma}_{0}$.

in the main text], we see two alternating peaks in both stress and pressure which are well correlated to the peaks in strain. Figure 10(a) shows how the relative viscosity varies with time at $\phi=0.76, \mu_{p}=0.4, \mathcal{G}=0.33$, and $\mathcal{F}=30$. Similar to Fig. 1(b) in the main text, we see that the "stress" viscosity $\eta_{\sigma}$ poorly represents the viscosity of the suspension and is closer to the peak viscosity, while $\eta_{|\dot{\gamma}|}$ is better in describing the suspension's viscosity. Figure 10(b) and (c) shows the instantaneous stress and pressure for the same case as in Fig. 10(a). While $\eta_{\sigma}$ is close to the peak of instantaneous viscosities, the corresponding stresses $\langle\sigma\rangle$ and pressures $\langle P\rangle$ do better in representing a suspension's average (signed) stress and pressure. Corresponding shear-rate average stresses $\sigma_{[\dot{\gamma} \mid}$ and pressures $P_{[\dot{\gamma} \mid}$ are also showed, both yielding a good representation of the (time-)averaged stresses and pressures.
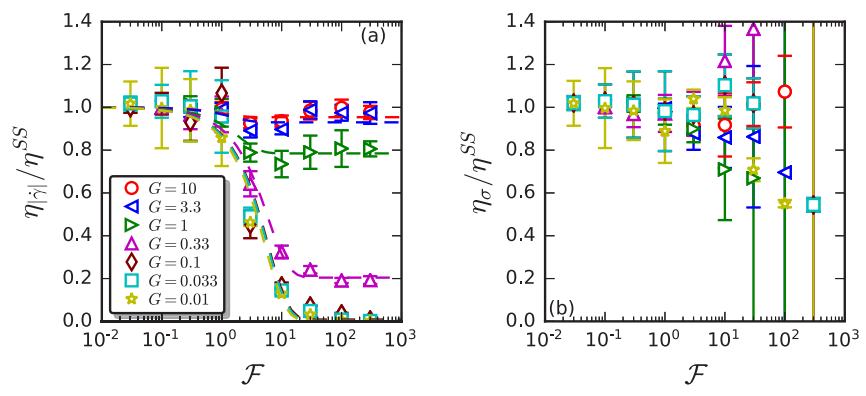

FIG. 11. (a) Reduced "dissipative" viscosities $\eta_{|\dot{\gamma}|} / \eta^{S S}$ at $\phi=$ 0.76 and (b) reduced "stress" viscosities $\eta_{\sigma} / \eta^{S S}$ as function of $\mathcal{F}$ at various $\mathcal{G}, \phi=0.76$, and $\mu_{p}=0.4$.

\section{APPENDIX B: COMPARISON BETWEEN "STRESS" VISCOSITIES $\eta_{\sigma} / \eta^{S S}$ AND "DISSIPATIVE" VISCOSITIES $\eta_{|\dot{\gamma}|} / \eta^{S S} \mathrm{AT} \phi=0.76$}

Figure 11 shows a comparison of using (a) "dissipative" viscosity $\eta_{|\dot{\gamma}|} / \eta^{S S}$ and (b) "stress" viscosity $\eta_{\sigma} / \eta^{S S}$. Figure 11(a) is the same as Fig. 2(a) in the main text. While $\eta_{\sigma} / \eta^{S S}$ is close to $\eta_{|\dot{\gamma}|} / \eta^{S S}$ at $\mathcal{F}<1$, it becomes scattered with large errors bars as $\mathcal{F}>1$. This is because $\sigma$ and $\dot{\gamma}$ change sign at $\mathcal{F}>1$ due to shear reversal. As $\mathcal{F} \rightarrow \infty$ we have $\langle\sigma\rangle \rightarrow 0$ and $\langle\dot{\gamma}\rangle \rightarrow 0$. Therefore, $\eta_{\sigma}$ is no longer a good measurement when $\mathcal{F}>1$.

\section{APPENDIX C: RESCALED NORMAL VISCOSITIES $\eta_{|\dot{\gamma}|}^{P} / \eta_{P}^{S S}$ AT $\phi=0.76$ FOR SUSPENSIONS OF FRICTIONAL PARTICLES}

In Fig. 12(a) we show rescaled normal viscosities $\eta_{|\dot{\gamma}|}^{P} / \eta_{P}^{S S}$ as function of $\mathcal{F}$ at $\phi=0.76$ and $\mu_{p}=0.4$. The parameters are exactly the same as in Fig. 2 in the main text. The normal viscosity is calculated from $\eta_{|\dot{\gamma}|}^{P}=\langle P\rangle_{|\dot{\gamma}|} /\langle\dot{\gamma}\rangle_{|\dot{\gamma}|}$ and $\eta_{P}^{S S}$ is the corresponding value at steady-shear. Similar as in Fig. 2, we find that the normal viscosities are close to steady-shear results as soon as $\mathcal{F}<1$ and decreases as $\mathcal{F}$ increases, well
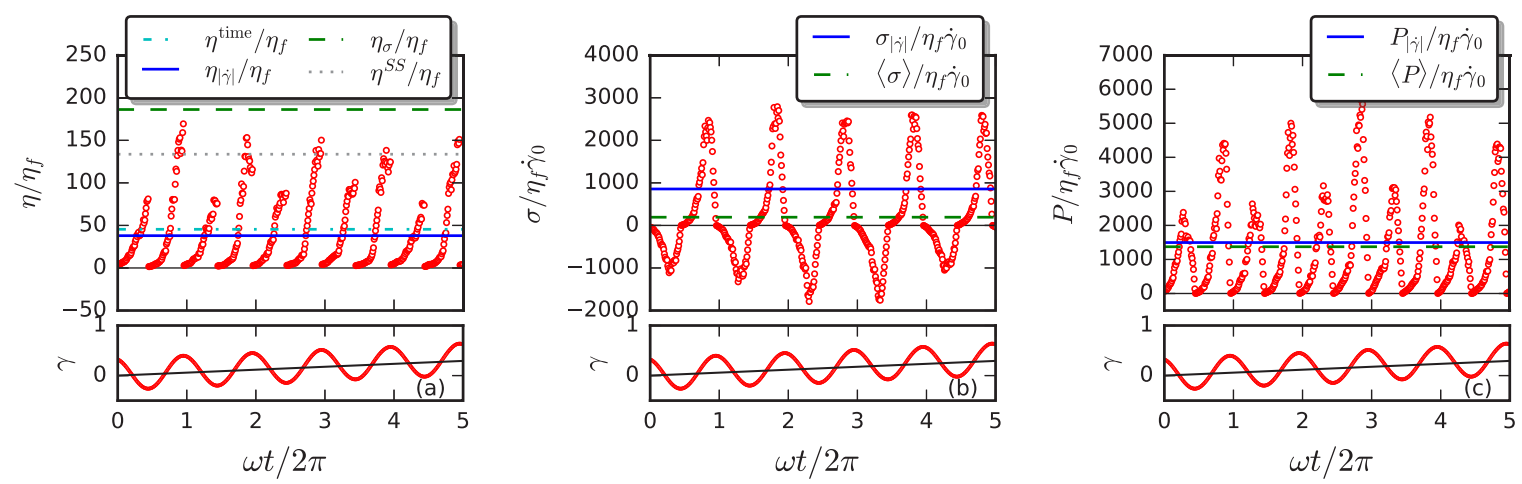

FIG. 10. (a) Instantaneous relative viscosities $\eta / \eta_{f}$, (b) instantaneous rescaled stresses $\sigma / \eta_{f} \dot{\gamma}_{0}$, and (c) instantaneous rescaled pressures $P / \eta_{f} \dot{\gamma}_{0}$ at $\phi=0.76, \mu_{p}=0.4, \mathcal{G}=0.33$, and $\mathcal{F}=30$ as function of time. In (a), lines give "stress" viscosity $\eta_{\sigma} / \eta_{f}$ (green-dashed), "dissipative" viscosity $\eta_{|\dot{\gamma}|} / \eta_{f}$ (solid-blue), and normal time-average $\eta^{\text {time }} / \eta_{f}$ (cyan-dash-dotted); grey dotted line indicates viscosity for steady shear (SS) flow at $\phi=0.76$ (i.e., $\mathcal{F}=0$ ), thin black solid line indicates zero line; in (b) and (c) green-dashed lines indicate values of $\langle\sigma\rangle / \eta_{f} \dot{\gamma}_{0}$ and $\langle P\rangle / \eta_{f} \dot{\gamma}_{0}$, respectively, and blue solid lines indicate the corresponding shear-rate-averaged quantities. The rectangular plots beneath the main plots illustrate how strain evolves in the same time span with black lines indicate how strain will evolve accounting only for the average shear-rate $\dot{\gamma}_{0}$. 

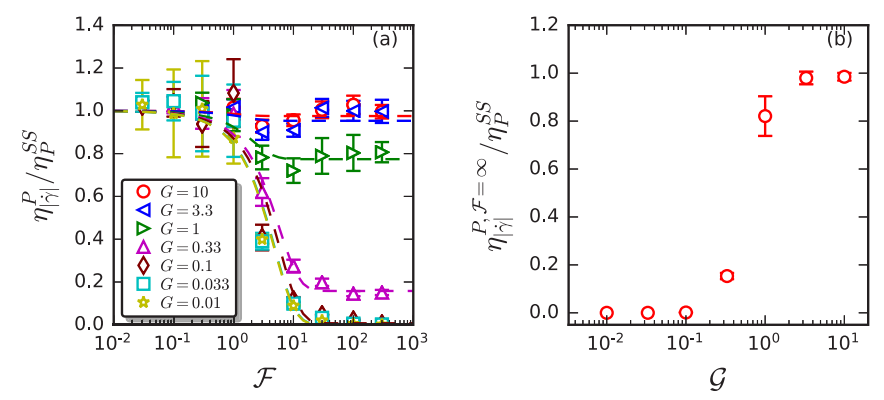

FIG. 12. (a) Reduced normal viscosities $\eta_{|\dot{\gamma}|}^{P} / \eta_{P}^{S S}$ as function of $\mathcal{F}$ at various $\mathcal{G}, \phi=0.76$, and $\mu_{p}=0.4 . \eta_{P}^{S S}$ is the normal viscosity at steady-shear at $\phi=0.76$. Dashed lines are best fits using a phenomenological hyperbolic tangent function $\eta_{|\dot{\gamma}|}^{P} / \eta_{P}^{S S}=1-$ $c_{1} \tanh \left(c_{2} \mathcal{F}\right)$, where $c_{1}=\left(1-\eta_{|\dot{\gamma}|}^{P, \mathcal{F}=\infty} / \eta_{P}^{S S}\right)$ and $c_{2}$ are two fitting parameters. (b) The reduced normal viscosity $\eta_{|\dot{\gamma}|}^{P} / \eta_{P}^{S S}$ as function of $\mathcal{G}$ at $\mathcal{F}=\infty$.

described by hyperbolic tangent functions. The decrease is small for large $\mathcal{G}$ 's while significant for small $\mathcal{G}$ 's. We illustrate this trend in Fig. 12(b) where we plot the normal viscosity at $\mathcal{F}=\infty$ as function of $\mathcal{G}$.

\section{APPENDIX D: "DISSIPATIVE" VISCOSITIES $\eta_{|\dot{y}|} / \eta^{s S,}$ NUMBER OF CONTACTS $Z_{|\dot{y}|}$ AT OTHER PACKING FRACTIONS $\phi$ FOR SUSPENSIONS OF FRICTIONAL PARTICLES, AND AT $\phi=0.76$ FOR SUSPENSIONS OF FRICTIONLESS PARTICLES}

Figure 13 shows how the strain-average viscosity $\eta_{|\dot{\gamma}|} / \eta^{S S}$ (a,b) and the number of contacts $Z_{|\dot{\gamma}|}(\mathrm{c}, \mathrm{d})$ vary with $\mathcal{F}$ for suspensions consisted of frictional particles at $\phi=0.67$ and $\phi=0.74$ and various $\mathcal{G}$. The observations are essentially the
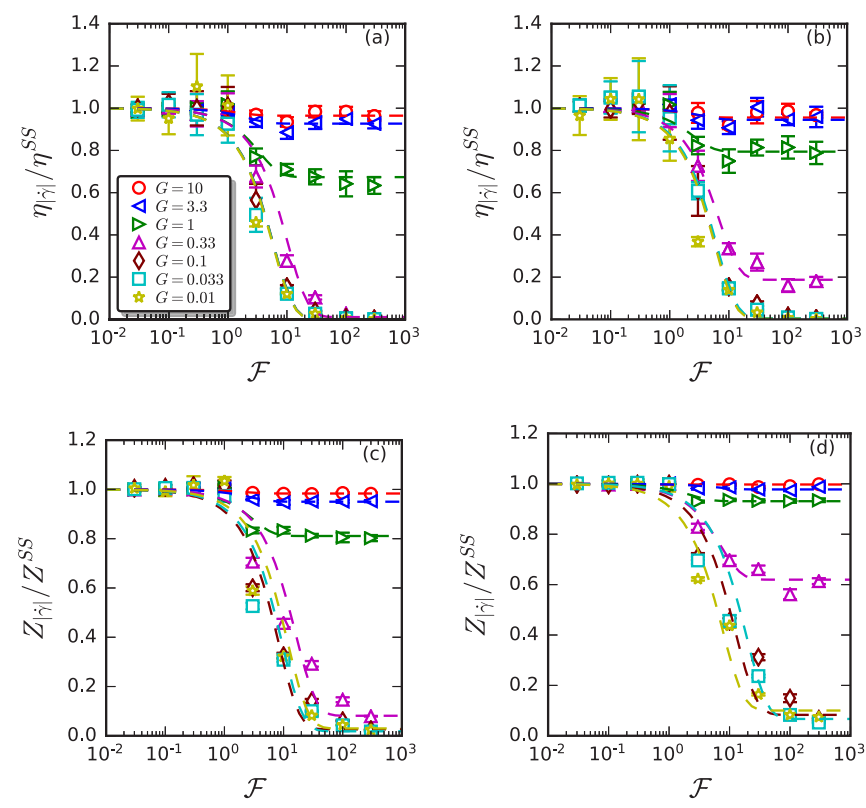

FIG. 13. Reduced viscosities $\eta_{|\dot{\gamma}|} / \eta^{S S}$ at (a) $\phi=0.67$, (b) $\phi=$ 0.74 ; and $Z_{|\dot{\gamma}|}$ at (c) $\phi=0.67$, (d) $\phi=0.74$; at various $\mathcal{G}$ and $\mu_{p}=$ 0.4 ; dashed lines are best fits using the hyperbolic tangent function introduced in Fig. 2 in the main text.
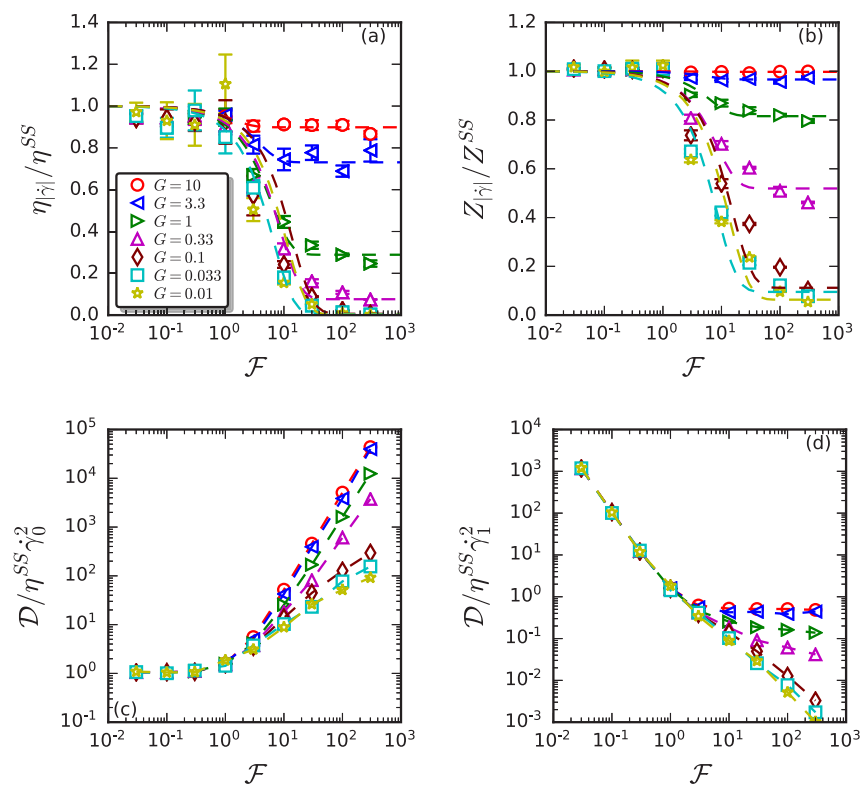

FIG. 14. (a) Reduced viscosities $\eta_{|\dot{\gamma}|} / \eta^{S S}$, (b) $Z_{|\dot{\gamma}|}$, (c) $\mathcal{D} / \dot{\gamma}_{0}^{2}$, and (d) $\mathcal{D} / \dot{\gamma}_{1}^{2}$ as function of $\mathcal{F}$ at various $\mathcal{G}, \phi=0.76$, and $\mu_{p}=0$; dashed lines in (a) and (b) are best fits using the hyperbolic tangent function introduced in Fig. 2 in the main text, and dashed lines in (c) and (d) are $\eta_{|\dot{\gamma}|} / \eta^{S S}\left(1+0.5 \mathcal{F}^{2}\right)$ and $\eta_{|\dot{\gamma}|} / \eta^{S S}\left(\frac{1}{\mathcal{F}^{2}}+0.5\right)$ with $\eta_{|\dot{\gamma}|}$ obtained from (a).

same seen in Fig. 2(a,b) in the main text, indicating that our observations in Fig. 2 are general for frictional suspensions at various $\phi$ 's. A minor difference between different $\phi$ is that at low packing fraction $\phi=0.67$, the viscosity and the number of contacts decrease to almost zero already at $\mathcal{G}=0.33$. Figure 14 shows how (a) the strain-average viscosity $\eta_{|\dot{\gamma}|} / \eta^{S S}$, (b) the number of contacts $Z_{|\dot{\gamma}|}$ and the dissipation $\mathcal{D}$ normalized by (c) $\dot{\gamma}_{0}^{2}$ and (d) $\dot{\gamma}_{1}^{2}$ vary with $\mathcal{F}$ at $\phi=0.76$ and $\mu_{p}=0$. Again, the results we have is almost identical to those seen in Fig. 2 .

\section{APPENDIX E: AN ALTERNATIVE DEFINITION OF $\mu_{|\dot{\gamma}|}$ AND $J_{|\dot{\gamma}|}$}

In Fig. 3 in the main text we showed $\mu(J)$ and $Z(J)$ with $\mu_{|\dot{\gamma}|}=\langle\sigma / P\rangle_{|\dot{\gamma}|}$ and $J_{|\dot{\gamma}|}=\eta_{f}\langle\dot{\gamma} / P\rangle_{|\dot{\gamma}|}$. Here, in Fig. 15 we show plots of $\mu(J)$ and $Z(J)$ using an alternative definition of $\mu_{|\dot{\gamma}|}$ and $J_{|\dot{\gamma}|}$, with $\mu_{|\dot{\gamma}|}=\sigma_{|\dot{\gamma}|} / P_{|\dot{\gamma}|}$ and $J_{|\dot{\gamma}|}=\eta_{f} \dot{\gamma}_{|\dot{\gamma}|} / P_{|\dot{\gamma}|}$. The parameters are exactly the same as in Fig. 3 in the main text. The main observations are the same as in Fig. 3, except the collapse being worse especially in Fig. 15(c) (corresponding to $Z$ ) at moderate $J_{|\dot{\gamma}|}$. Another difference is that the decrease of $\mu_{|\dot{\gamma}|}$ at $J_{|\dot{\gamma}|} \gg J^{S S}(\phi)$ is much less significant compared with Fig. 3. In Fig. 15(a) $\mu_{|\dot{\gamma}|}$ seems more likely to be saturating rather than decreasing.

\section{APPENDIX F: TIME SERIES OF $\phi$ FOR PURE OSCILLATORY FLOWS UNDER CONSTANT PRESSURE CLOSE TO $\phi_{c}$}

Figure 16 shows time series of $\phi-\phi_{|\dot{\gamma}|}$ for pure oscillatory flows under constant pressure (see Fig. 4 in the main text). 

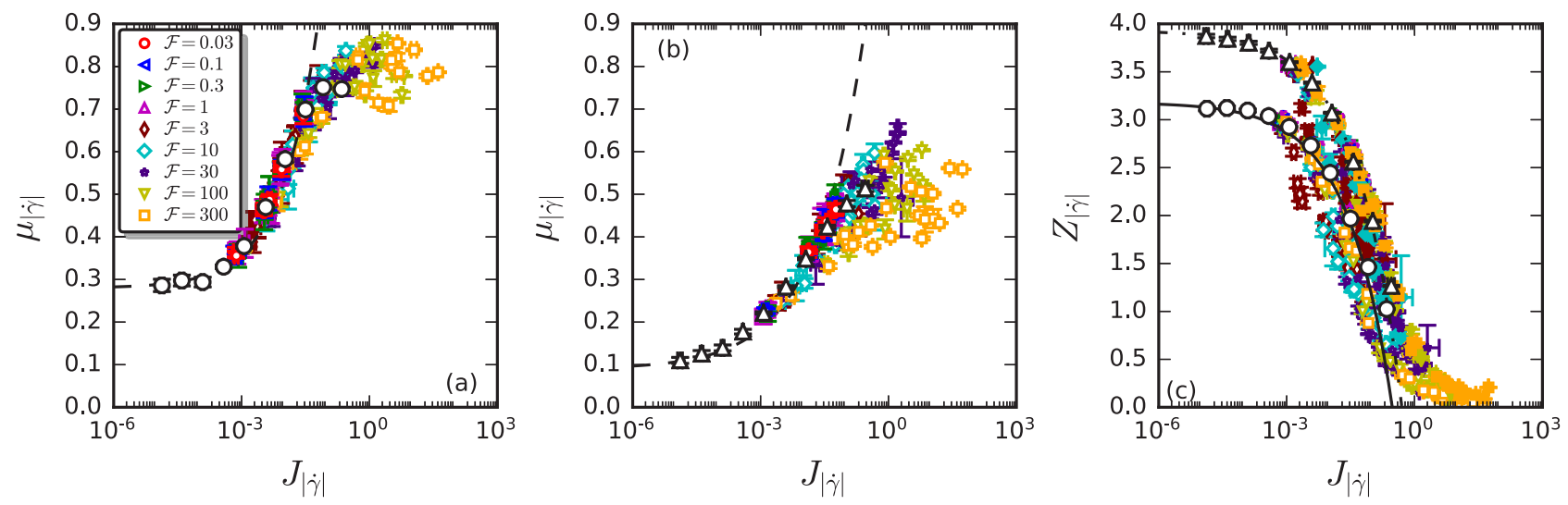

FIG. 15. Flow curves with extended $\mu(J)$ rheology for (a) $\mu_{|\dot{\gamma}|}$ for suspensions with frictional particles $\left(\mu_{p}=0.4\right)$, (b) $\mu_{|\dot{\gamma}|}$ for suspensions with frictionless particles $\left(\mu_{p}=0\right)$, and (c) $Z$ for both suspensions composed of either frictional (empty symbols) or frictionless particles (filled symbols) using $\mu_{|\dot{\gamma}|}=\sigma_{|\dot{\gamma}|} / P_{|\dot{\gamma}|}$ and $J_{|\dot{\gamma}|}=\eta_{f} \dot{\gamma}_{|\dot{\gamma}|} / P_{|\dot{\gamma}|}$. Black symbols corresponds to steady-shear values [25] for (circles) frictional and (triangles) frictionless particles, lines according to [25]. Various symbols and colors in (a) and (b) corresponds to various $\mathcal{F}$ as indicated in legends, with $\phi \in[0.67,0.79]$ for frictional and $\phi \in[0.72,0.82]$ for frictionless particles and $\mathcal{G} \in\left[10^{-2}, 10\right]$.

(a) is for suspensions of frictional particles with $\phi_{|\dot{\gamma}|}^{\mathcal{G}=0.33}=$

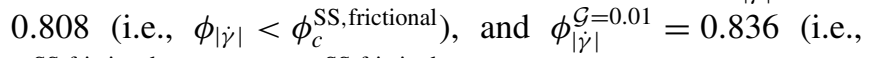
$\phi_{c}^{\text {SS, frictional }}<\phi_{|\dot{\gamma}|}<\phi_{c}^{\text {SS, frictionless }}$ ); (b) is for suspensions of frictionless particles with $\phi_{|\dot{\gamma}|}^{\mathcal{G}=0.33}=0.831$ and $\phi_{|\dot{\gamma}|}^{\mathcal{G}=0.01}=$ 0.838 , both have $\phi_{c}^{\text {SS, frictional }}<\phi_{|\dot{\gamma}|}<\phi_{c}^{\text {SS, frictionless }}$. For both frictional and frictionless cases, $\phi$ clearly fluctuate around $\phi_{|\dot{\gamma}|}$ with large fluctuations for the larger $\mathcal{G}$ value. Figure 16(c) shows the evolution over 100 periods for the $\mathcal{G}=0.01, \mu_{p}=$ 0.4 , and $\phi_{|\dot{\gamma}|}=0.836$. We see that $\phi$ remains stable over 100 periods.

\section{APPENDIX G: COMPARISON WITH RESULTS CONSIDERING LUBRICATION FORCES}

In this section we show some results with lubrication forces and compare them with the results without lubrication force. The lubrication force between each pair of disks is calculated as

$$
\begin{gathered}
\mathbf{F}_{\text {lub }, \mathrm{n}}^{i j}=\left[-\frac{3}{8} \pi \eta_{f} d_{i j} \frac{\left(\mathbf{V}_{i}-\mathbf{V}_{j}\right) \cdot \mathbf{n}_{i j}}{h_{i j}+\delta}\right] \mathbf{n}_{i j}, \\
\mathbf{F}_{\text {lub }, \mathrm{t}}^{i j}=\left[-\frac{1}{2} \pi \eta_{f} \ln \left(\frac{d_{i j}}{2\left(h_{i j}+\delta\right)}\right)\left(\mathbf{V}_{i}-\mathbf{V}_{j}\right) \cdot \mathbf{t}_{i j}\right] \mathbf{t}_{i j},
\end{gathered}
$$

where Eq. (G1) gives the normal component and Eq. (G2) gives the tangential component, $h_{i j}$ is the gap between two disks $i$ and $j, \mathbf{V}_{i}$ and $\mathbf{V}_{j}$ are the velocity of disk $i$ and $j$ at the points of closest distance (i.e., a linear combination of the translational and rotational velocities of the discs), $d_{i j}=$ $2 d_{i} d_{j} /\left(d_{i}+d_{j}\right)$, the regularization length $\delta=0.05 d$ or $0.01 d$, where $d$ being the average disk diameter. For more detailed description of lubrication forces see [2]. Figure 17 shows (a) the "dissipative" viscosity $\eta_{|\dot{\gamma}|} / \eta^{S S}$, (b) the number of contacts $Z_{|\dot{\gamma}|}$, and the dissipation $\mathcal{D}$ normalized by (c) $\dot{\gamma}_{0}^{2}$ and (d) $\dot{\gamma}_{1}^{2}$ at various $\mathcal{G}$ with lubrication force at $\delta=0.05 d$. The figure is the counterpart to Fig. 2 from the main text (i.e., all parameters are identical to the main text except the lubrication forces).
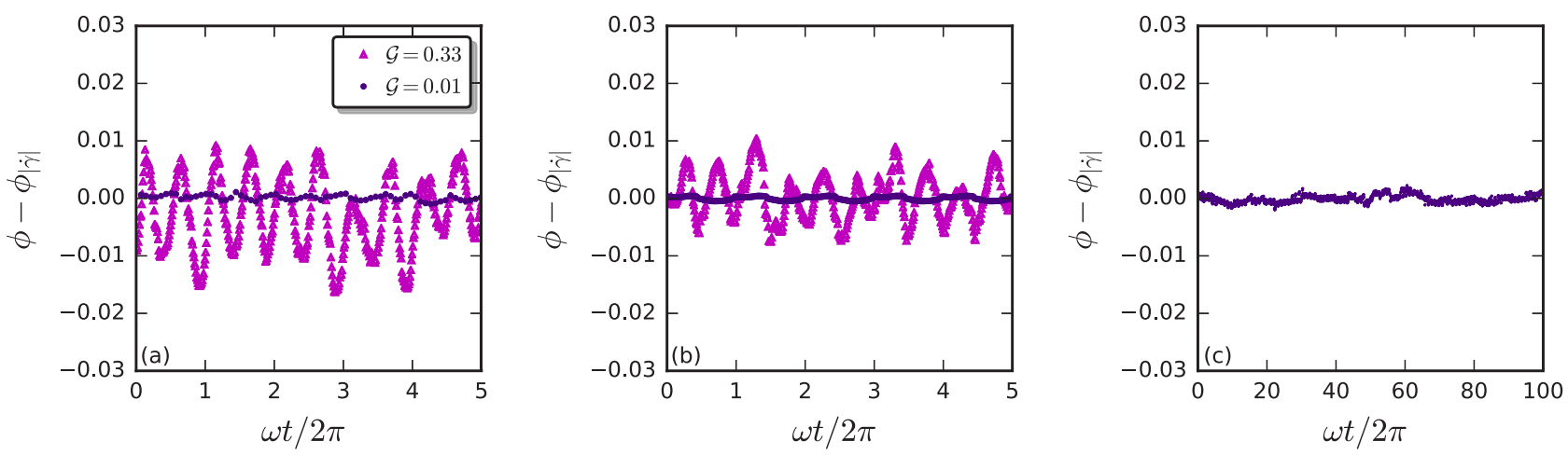

FIG. 16. Time series of $\phi-\phi_{|\dot{\gamma}|}$ for pure oscillatory flows $(\mathcal{F}=\infty)$ under constant pressure close to $\phi^{c}\left(J_{|\dot{\gamma}|} \sim 10^{-3}\right)$ for (a) suspensions of frictional particles and (b) suspensions of frictionless particles at $\mathcal{F}=\infty, \mathcal{G}=0.33$, and 0.01 ; (c) the same case as in (a) at $\mathcal{G}=0.01$ but over 100 oscillation periods. 

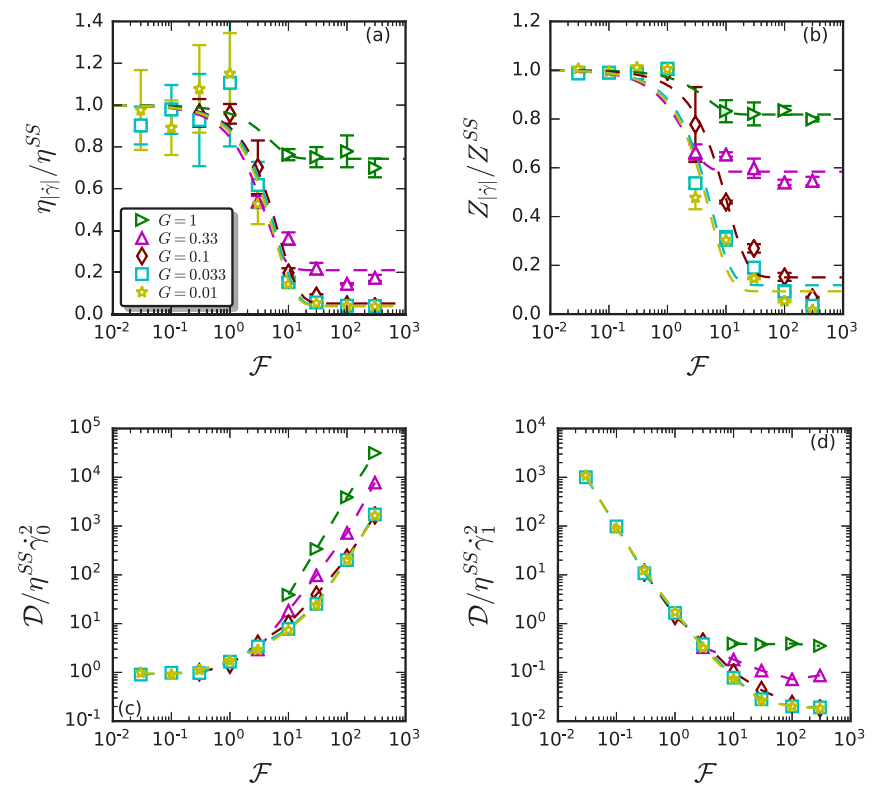

FIG. 17. (a) Reduced viscosities $\eta_{|\dot{\gamma}|} / \eta^{S S}$, (b) $Z_{|\dot{\gamma}|}$, (c) $\mathcal{D} / \dot{\gamma}_{0}^{2}$, and (d) $\mathcal{D} / \dot{\gamma}_{1}^{2}$ as function of $\mathcal{F}$ at various $\mathcal{G}, \phi=0.76$, and $\mu_{p}=0.4$ with lubrication forces at $\delta=0.05 d$; dashed lines in (a) and (b) are best fits using the phenomenological hyperbolic tangent function introduced in Fig. 2 in the main text, and dashed lines in (c) and (d) are $\eta_{|\dot{\gamma}|} / \eta^{S S}\left(1+0.5 \mathcal{F}^{2}\right)$ and $\eta_{|\dot{\gamma}|} / \eta^{S S}\left(\frac{1}{\mathcal{F}^{2}}+0.5\right)$ with $\eta_{|\dot{\gamma}|}$ obtained from (a); values of $\eta^{S S}$ and $Z^{S S}$ are obtained from steady shear with lubrication.

Similar observations are found, with viscosities close to steady shear ones at small $\mathcal{F}$ 's which then drops as $\mathcal{F}$ increases. The drop in viscosity is more substantial for small $\mathcal{G}$, just as in the case without any lubrication forces. The main difference is that for small $\mathcal{G}$ 's the viscosity decreases to a finite value (approximately 5\% of $\eta^{S S}$ ) compared to a value close to zero in the case of having no lubrication forces. In Fig. 18 we compares simulation results without lubrication with results including lubrication force using two $\delta, 0.05 d$ and $0.01 d$ at $\mathcal{G}=0.01$, and $\phi=0.76$. We can see that viscosity decreased substantially for all three curves, except that the viscosity decrease to a finite value for cases with lubrication
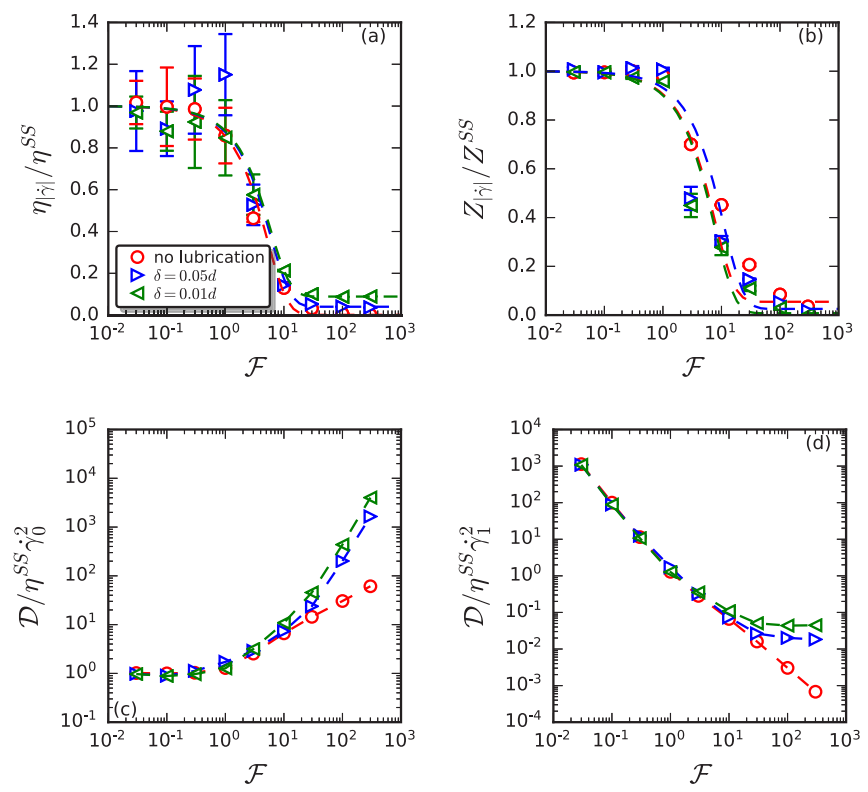

FIG. 18. Comparisons between having no lubrication forces and having lubrication forces with $\delta=0.05 d$ and $\delta=0.01 d$ for (a) reduced viscosities $\eta_{|\dot{\gamma}|} / \eta^{S S}$, (b) $Z_{|\dot{\gamma}|}$, (c) $\mathcal{D} / \dot{\gamma}_{0}^{2}$, and (d) $\mathcal{D} / \dot{\gamma}_{1}^{2}$ at $\mathcal{G}=$ $0.01, \phi=0.76$, and $\mu_{p}=0.4$; dashed lines in (a) and (b) are best fits using the phenomenological hyperbolic tangent function introduced in Fig. 2 in the main text, and dashed lines in (c) and (d) are $\eta_{|\dot{\gamma}|} / \eta^{S S}\left(1+0.5 \mathcal{F}^{2}\right)$ and $\eta_{|\dot{\gamma}|} / \eta^{S S}\left(\frac{1}{\mathcal{F}^{2}}+0.5\right)$ with $\eta_{|\dot{\gamma}|}$ obtained from (a); values of $\eta^{S S}$ and $Z^{S S}$ are obtained from corresponding steady shear.

compared to zero for the case without lubrication forces. And the plateau is slightly higher for $\delta=0.01 d$ (approximately $8 \%$ of $\eta^{S S}$ ). The difference in viscosities are clearer seen if one looks at the dissipation rates instead. At $\mathcal{F}>10$, the dissipation rate for the case with lubrication diverge from the case without lubrications, in (c) a larger increasing slope and (d) reaching plateau, both reflecting a finite viscosity at $\mathcal{F} \rightarrow \infty . Z_{|\dot{\gamma}|}$ at $\mathcal{F}>10$, on the other hand, does not show noticeable difference, indication that the difference in viscosity and dissipation is a result from solely lubrication forces.
[1] G. Chatté, J. Comtet, A. Niguès, L. Bocquet, A. Siria, G. Ducouret, F. Lequeux, N. Lenoir, G. Ovarlez, and A. Colin, Shear thinning in non-Brownian suspensions, Soft Matter 14, 879 (2018).

[2] M. Trulsson, B. Andreotti and P. Claudin, Transition from the Viscous to Inertial Regime in Dense Suspensions, Phys. Rev. Lett. 109, 118305 (2012).

[3] C. Ness and J. Sun, Flow regime transitions in dense non-Brownian suspensions: Rheology, microstructural characterization, and constitutive modeling, Phys. Rev. E 91, 012201 (2015).

[4] R. Seto, R. Mari, J. F. Morris, and M. M. Denn, Discontinuous Shear Thickening of Frictional Hard-Sphere Suspensions, Phys. Rev. Lett. 111, 218301 (2013).
[5] M. Wyart and M. E. Cates, Discontinuous Shear Thickening without Inertia in Dense non-Brownian Suspensions, Phys. Rev. Lett. 112, 098302 (2014).

[6] S. Jamali and J. F. Brady. Alternative Frictional Model for Discontinuous Shear Thickening of Dense Suspensions: Hydrodynamics, Phys. Rev. Lett. 123, 138002 (2019).

[7] J. Dong and M. Trulsson, Analog of discontinuous shear thickening flows under confining pressure, Phys. Rev. Fluids 2, 081301(R) (2017).

[8] D. Bi, J. Zhang, B. Chakraborty, and R. P. Behringer, Jamming by shear, Nature 480, 355 (2011).

[9] P. Olsson and S. Teitel, Critical Scaling of Shear Viscosity at the Jamming Transition, Phys. Rev. Lett. 99, 178001 (2007). 
[10] B. Andreotti, J.-L. Barrat, and C. Heussinger, Shear Flow of non-Brownian Suspensions Close to Jamming, Phys. Rev. Lett. 109, 105901 (2012).

[11] F. Boyer, E. Guazzelli and O. Pouliquen, Unifying Suspension and Granular Rheology, Phys. Rev. Lett. 107, 188301 (2011)

[12] L. E. Silbert, Jamming of frictional spheres and random loose packing, Soft Matter 6, 2918-2924 (2010).

[13] A. Donev, I. Cisse, D. Sachs, E. A. Variano, F. H. Stillinger, R. Connelly, S. Torquato, and P. M. Chaikin, Improving the density of jammed disordered packings using ellipsoids, Science 303, 990 (2004).

[14] E. Azéma, F. Radjai, and J.-N. Roux, Internal friction and absence of dilatancy of packings of frictionless polygons, Phys. Rev. E 91, 010202(R) (2015).

[15] D. B. Nagy, P. Claudin, T. Börzsönyi, and E. Somfai, Rheology of dense granular flows for elongated particles, Phys. Rev. E 96, 062903 (2017).

[16] M. Trulsson, Rheology and shear jamming of frictional ellipses, J. Fluid Mech. 849, 718 (2018).

[17] T. Marschall, Y.-E. Keta, P. Olsson, and S. Teitel, Orientational Ordering in Athermally Sheared, Aspherical, Frictionless Particles, Phys. Rev. Lett. 122, 188002 (2019).

[18] T. A. Marschall and S. Teitel, Shear-driven flow of athermal, frictionless, spherocylinder suspensions in two dimensions: Stress, jamming, and contacts, Phys. Rev. E 100, 032906 (2019).

[19] K. M. Salerno, D. S. Bolintineanu, G. S. Grest, J. B. Lechman, S. J. Plimpton, I. Srivastava, and L. E. Silbert, Effect of shape and friction on the packing and flow of granular materials, Phys. Rev. E 98, 050901(R) (2018).

[20] E. Irani, P. Chaudhuri, and C. Heussinger, Impact of Attractive Interactions on the Rheology of Dense Athermal Particles, Phys. Rev. Lett. 112, 188303 (2014).

[21] N. Berger, E. Azéma, J.-F. Douce, and F. Radjai, Scaling behavior of cohesive granular flows, Europhys. Lett. 112, 64004 (2015).

[22] M. Trulsson, M. Bouzid, J. Kurchan, E. Clément, P. Claudin and B. Andreotti, Athermal analog of sheared dense Brownian suspensions, Europhys. Lett. 111, 18001 (2015).
[23] N. J. Wagner and J. F. Brady, Shear thickening in colloidal dispersions, Phys. Today 62(10), 27 (2009).

[24] C. Ness and J. Sun, Shear thickening regimes of dense nonBrownian suspensions, Soft Matter 12, 914 (2016).

[25] J. Dong and M. Trulsson, Unifying viscous and inertial regimes of discontinuous shear thickening suspensions, J. Rheology 64 , 255 (2020).

[26] P. Sehgal, M. Ramaswamy, I. Cohen, B. J. Kirby, Using Acoustic Perturbations to Dynamically Tune Shear Thickening in Colloidal Suspensions, Phys. Rev. Lett. 123, 128001 (2019).

[27] N. Y. C. Lin, C. Ness, M. E. Cates, J. Sun, and I. Cohen, Tunable shear thickening in suspensions, Proc. Natl. Acad. Sci. USA 113, 10774 (2016).

[28] C. Ness, R. Mari, and M. E. Cates, Shaken and stirred: Random organization reduces viscosity and dissipation in granular suspensions, Sci. Adv. 4, eaar3296 (2018).

[29] D. J. Pine, J. P. Gollub, J. F. Brady and A. M. Leshansky, Chaos and threshold for irreversibility in sheared suspensions, Nature 438, 997 (2005).

[30] R. H. Ewoldt, A. E. Hosoi, and G. H. McKinley, New measures for characterizing nonlinear viscoelasticity in large amplitude oscillatory shear, J. Rheol. 52, 1427 (2008).

[31] M. Trulsson, E. DeGiuli, and M. Wyart, Effect of friction on dense suspension flows of hard particles, Phys. Rev. E 95, 012605 (2017).

[32] G. Düring, D. Bartolo, and J. Kurchan, Irreversibility and selforganization in hydrodynamic echo experiments, Phys. Rev. E 79, 030101(R) (2009).

[33] R. Jeanneret and D. Bartolo, Geometrically protected reversibility in hydrodynamic Loschmidt-echo experiments, Nat. Commun. 5, 3474 (2014).

[34] D. Ishima and H. Hayakawa, Scaling laws for frictional granular materials confined by constant pressure under oscillatory shear, Phys. Rev. E 101, 042902 (2020).

[35] E. Rojas, M. Trulsson, B. Andreotti, E. Clément, and R. Soto, Relaxation processes after instantaneous shear-rate reversal in a dense granular flow, Europhys. Lett. 109, 64002 (2015). 